\title{
Oecologia
}

July 2013, Volume 172, Pages 631-643

http://dx.doi.org/10.1007/s00442-012-2527-1

(c) Springer-Verlag Berlin Heidelberg 2012

The original publication is available at http://www.springerlink.com

\section{Estimating age at maturation and energy-based life-history traits from individual growth trajectories with nonlinear mixed-effects models}

\author{
Thomas Brunel ${ }^{1,{ }^{*}}$, Bruno Ernande ${ }^{2}$, Fabian M. Mollet ${ }^{1,3}$, Adriaan D. Rijnsdorp ${ }^{1,4}$ \\ ${ }^{1}$ Wageningen IMARES, P.O. Box 68, 1970 AB, IJmuiden, The Netherlands \\ 2 IFREMER, Laboratoire Ressources Halieutiques, 150 quai Gambetta, BP 699, 62321, Boulogne-sur-Mer, \\ France \\ ${ }^{3}$ Blueyou Consulting Ltd., Zentralstrasse 156, 8003, Zürich, Switzerland \\ 4 Aquaculture and Fisheries Group, Wageningen University, P.O. Box 338, 6700 AH, Wageningen, The \\ Netherlands \\ *: Corresponding author : Thomas Brunel, email address : thomas.brunel@wur.nl
}

\begin{abstract}
:
A new method is presented to estimate individuals' (1) age at maturation, (2) energy acquisition rate, (3) energy expenditure for body maintenance, and (4) reproductive investment, and the multivariate distribution of these traits in a population. The method relies on adjusting a conceptual energy allocation model to individual growth curves using nonlinear mixed-effects modelling. The method's performance was tested using simulated growth curves for a range of life-history types. Individual age at maturation, energy acquisition rate and the sum of maintenance and reproductive investment rates, and their multivariate distribution, were accurately estimated. For the estimation of maintenance and reproductive investment rates separately, biases were observed for life-histories with a large imbalance between these traits. For low reproductive investment rates and high maintenance rates, reproductive investment rate estimates were strongly biased whereas maintenance rate estimates were not, the reverse holding in the opposite situation. The method was applied to individual growth curves back-calculated from otoliths of North Sea plaice (Pleuronectes platessa) and from scales of Norwegian spring spawning herring (Clupea harengus). For plaice, maturity ogives derived from our individual estimates of age at maturation were almost identical to the maturity ogives based on gonad observation in catch samples. For herring, we observed $51.5 \%$ of agreement between our individual estimates and those directly obtained from scale reading, with a difference lower than 1 year in $97 \%$ of cases. We conclude that the method is a powerful tool to estimate the distribution of correlated lifehistory traits for any species for which individual growth curves are available.
\end{abstract}

Keywords: Bioenergetics growth model ; Individual growth trajectory ; Life-history trade-offs ; Energy acquisition ; Maintenance ; Reproductive investment ; Sexual maturation 


\section{Introduction}

Phenotypic variation in age and size at maturation is of central interest in evolutionary ecology (see reviews in Bernardo (1993), Berrigan and Charnov (1994) and Stearns and Koella (1986)) because of their effects on several fitness components (Stearns and Hoekstra 2005). As for any trait, it may result from genetic variation and/or phenotypic plasticity (Bernardo 1994; Stearns 1984). Genetic variation may occur among populations, among cohorts within populations, and within cohorts (Bernardo 1994; Johnson 2001; Reznick 1990; Stearns 1984). Phenotypic plasticity may occur in response to variability in environmental factors, such as food level (Berrigan and Charnov 1994) and temperature (Atkinson 1994), and to density-dependent processes (Engelhard and Heino 2004), affecting somatic growth and/or the maturation process itself (Bernardo 1993; Dhillon and Fox 2004; Kuparinen et al. 2011; Stearns and Koella 1986). Additionally, age and size at maturation will likely covary with growth rate and reproductive investment, and covariation in these traits might also affect fitness. The analysis of variation in age and size at maturation and covarying life-history traits is hampered by the difficulty to collect data on individual organisms in the wild. Consequently, most studies on variation in growth rate, the onset of sexual maturation and reproductive investment have focused on the population level in the wild or the individual level under experimental conditions.

For many animal taxa, information on individuals' growth history is stored in their calcified structures, such as otoliths and scales in fish, shells in molluscs, endoskeleton in echinoderms, exoskeleton in coral, skeleton in reptiles and amphibians, and teeth in mammals (Campana (2001) and references therein). If the relationship between the size of calcified structures and body size is known, individuals' growth curves can be back-calculated from the distance between successive annual rings in the calcified structures. Estimating individuals' traits from 
the analysis of back-calculated individual growth curves could then compensate for the lack of direct individual data in wild populations.

It has been proposed that individual age and size at maturation can be estimated using segmented regression to detect a "breakpoint" in individual growth trajectories (Baulier and Heino 2008; Rijnsdorp and Storbeck 1995). This breakpoint corresponds to a decrease in somatic growth rate that takes place after maturation as a result of the energy allocation tradeoff between reproduction and somatic growth (Roff 1983). Explicit energy allocation models, which derive somatic growth rate as resulting from the balance between energy acquisition and energy expenditures for body maintenance and reproduction, can provide a useful framework to extract more precise and more complete life-history information from backcalculated growth data. Recently, Mollet et al. (2010) fitted an energy allocation-based growth model to individual growth curves back-calculated from fish otoliths in order to estimate age at maturation and the rates of energy acquisition and energy expenditures for maintenance and reproductive investment (hereafter referred to as acquisition rate, maintenance rate and reproductive rate). However, this method, which consists in fitting the model separately for each individual, had some weaknesses. First, the estimates of maintenance rate and reproductive rate were confounded: one was frequently estimated at zero, while the other was estimated as the sum of the two actual rates. The authors overcame this problem either by discarding retrospectively confounded estimates or by assuming that maintenance was constant across individuals. Second, as they are calculated a posteriori from the collection of individual trait estimates, the characteristics (means and (co-)variances) of the traits' distribution in the population are sensitive to any error in individual estimates, including confounding. Third, the method was over-parameterized, 4 parameters being estimated based on 6 to 12 weight-at-age data points depending on individuals. 
In the present paper, nonlinear mixed-effects (NLME) modelling is used for fitting an energy allocation model to a set of individual growth curves at once, instead of separately for each individual as done in Mollet et al. (2010). This method first estimates the population level multivariate distribution (means and (co-)variances) of the parameters of interest (assuming they follow a multivariate normal distribution among individuals) and then predicts the individual parameters based on this population level distribution. Hence the population level estimates are not affected by errors in individual estimates and sample properties are naturally extrapolated to the population level. Moreover, the traits are constrained to be normally distributed. Bimodality in the distribution of maintenance and reproductive rates due to confounding as observed in Mollet et al. (2010) is hence avoided. Finally, the NLME framework relies on a likelihood approach which offers the possibility to obtain confidence intervals for the trait means and (co-)variances and to draw statistical inferences (e.g. testing for differences in parameter means between cohorts), which was not possible with Mollet et al.'s method.

In order to evaluate its performance for different types of life histories, the method is first applied to simulated datasets generated from varying combinations of life-history parameters. Performance is assessed by the accuracy of the estimates of individual parameters and their population distribution characteristics. In a second step, the method is applied to real backcalculated growth curves of Norwegian spring spawning herring (Clupea harengus) and North Sea plaice (Pleuronectes platessa) (hereafter NSSH and NSP, respectively). The resulting estimates of individual age at maturation and the corresponding population distribution are compared to independent estimates of maturation for each population, i.e. maturity ogives - the proportion of mature individuals at age, also interpreted as the mean probability of being mature at age - for NSP and individuals' age at first spawning estimated by scale reading for NSSH. 


\section{Material and methods}

\section{Traits estimation procedure}

122 Energy allocation-based growth model

123 Energy allocation models are based on fundamental biological processes: somatic growth rate

124 is determined by the balance between the acquisition of energy and its utilization for body 125 maintenance and, after maturation, for reproduction. The energy allocation model 126 corresponding to this process of somatic growth is expressed for juveniles and adults as 127 (Ricklefs 2003) :

$128 d m / d t= \begin{cases}a m^{\alpha}-b m^{\beta} & \text { for } t \leq t_{\mathrm{mat}} \\ a m^{\alpha}-b m^{\beta}-c m^{\gamma} & \text { for } t>t_{\mathrm{mat}}\end{cases}$

129 where $m$ is body mass, $t$ is time, $t_{\text {mat }}$ is age at maturation and the coefficients $a, b$ and $c$ 130 are the size-specific rates of energy acquisition, and energy use for body maintenance 131 reproduction, respectively.

132 For the purpose of illustration, we follow metabolic theory of ecology (West et al. 2001) in 133 choosing $\alpha=3 / 4$ and $\beta=\gamma=1$ (see Electronic Supplementary Material S1). Body mass as a 134 function of time is then given for juveniles $m_{J}(t)$ and adults $m_{A}(t)$ by

135

$$
\begin{array}{ll}
m_{J}(t)=\left(\frac{a}{b}-\left(\frac{a}{b}-m_{0}^{\frac{1}{4}}\right) \mathrm{e}^{-\frac{b}{4} t}\right)^{4} & \text { for } t \leq t_{\text {mat }} \\
m_{A}(t)=\left(\frac{a}{b+c}-\left(\frac{a}{b+c}-m_{\text {mat }}^{\frac{1}{4}}\right) \mathrm{e}^{-\frac{b+c}{4}\left(t-t_{\text {mat }}\right)}\right)^{4} & \text { for } t>t_{\text {mat }}
\end{array}
$$


136 where $m_{0}$ is body mass at birth and $m_{\text {mat }}=m_{J}\left(t_{\text {mat }}\right)$ is body mass at maturation. Both

137 juvenile and adult growth curves are sigmoid, with asymptotic body mass $m_{\infty J}=(a / b)^{4}$ and

$138 m_{\infty A}=(a /(b+c))^{4}$, respectively. After birth, an individual first follows the juveniles curve. It

139 switches to the adult curve at maturation (Fig. 1), after which an additional energy

140 expenditure for reproduction applies. The Heaviside step function, $H(\cdot)(H(x)=0$ for $x<0$

141 and $H(x)=1$ for $x \geq 0$ ) is used to combine juvenile and adult growth in a single function for

142 lifetime growth :

143

$m(t)=\left(1-H\left(t-t_{\text {mat }}\right)\right) m_{J}(t)+H\left(t-t_{\text {mat }}\right) m_{A}(t)$.

$145 \quad$ Statistical model

146 In order to estimate age at maturation and size-specific acquisition, maintenance and 147 reproductive rates, the energy allocation model $m(t)$ was fitted to cohort-wise sets of

148 individual growth curves using NLME modelling. NLME models are commonly used to fit

149 nonlinear parametric functions to longitudinal data, i.e. repeated measures over time on the

150 same subjects similar to the weight data describing individual growth curves here (Pinheiro

151 and Bates 2000). NLME models primarily estimate the distribution of the parameters in the

152 population - mean values and (co-)variances - and treat each individual as a sample from this

153 distribution. In practice, NLME model represent each individual parameter as the sum of a

154 fixed effect - the population mean of the parameter - and a random effect - the individual

155 parameter's deviation from the mean. The random effects of the different parameters are

156 constrained to follow a multivariate normal distribution centred on 0 . The algorithm first

157 estimates the population level distribution characteristics (parameters' means, i.e. fixed

158 effects, and (co-)variances) and predicts the individual parameters (random effects) 
159 afterwards, based on this distribution (but for the sake of simplicity, we will refer to 160 individual values as “estimates”).

161 NLME models were fitted using the nlme package (Pinheiro et al. 2007) of the statistical

162 software R (R Development Core Team 2006). Because of convergence problems when

163 estimating more than 4 parameters, only age at maturation and the 3 size-specific energy rates

164 were estimated, while we set initial body mass to a fixed value equal to its population

165 average. ). Confidence intervals of parameter estimates were produced by bootstrapping (1000

166 replicates) for NSP only as combining NLME and bootstrapping is computationally very

167 intensive. For further details on NLME models and on the fitting procedure used, the reader

168 can refer to Electronic Supplementary Material S2.

\section{Data simulation}

170 The method's performance was evaluated using simulated growth curves datasets

171 representing various life-history types. The 5 free parameters of the energy allocation model

172 (eq. 2) have the dimensions of mass $\left(m_{0}\right)$, time $\left(t_{\mathrm{mat}}\right)$, $\operatorname{time}^{-1}(b$ and $c)$ and mass ${ }^{1 / 4} \times \operatorname{time}^{-1}($

$173 \quad a$ ). These parameters can be combined into 3 dimensionless parameters:

$$
q=\frac{c}{b+c}=1-\frac{b}{b+c}, \quad \tau_{\mathrm{mat}}=t_{\mathrm{mat}}(b+c) \quad \text { and } v_{0}=m_{0}\left(\frac{b+c}{a}\right)^{4}=\frac{m_{0}}{m_{\infty A}}
$$

175 so that by varying only $q, \tau_{\text {mat }}$, and $v_{0}$ or, equivalently, by setting $1 /(b+c)$ and $a /(b+c)$ constant (which amounts to fix the sum $b+c$ and $a$ ) while varying $c, t_{\text {mat }}$ and $m_{0}$, the full

177 range of the model's parameter space can be investigated.

178 Several datasets of individual growth curves were simulated for different types of life-history 179 strategies by varying mean relative reproductive investment ( $\bar{q}$ taking values in $180\{1 / 5 ; 1 / 3 ; 1 / 2 ; 2 / 3 ; 4 / 5\})$, mean age at maturation $\left(\bar{t}_{\text {mat }}\right.$ varying between 4 and 8 years in 
181 steps of 1 year) and initial body mass $m_{0}$ (taking values in $\left\{0.5 \times 10^{-4} ; 10^{-4} ; 1.5 \times 10^{-4}\right\}(\mathrm{kg})$ ),

182 while keeping mean size-specific acquisition rate $\bar{a}$ and the sum of mean size-specific 183 maintenance and reproductive rates $\bar{b}+\bar{c}$ constant.

184 For each dataset corresponding to a given combination $\left\{\bar{q}, \bar{t}_{\text {mat }, m_{0}}\right\}$, 250 individual growth 185 curves were then generated according to the growth model (3) by sampling randomly 186 individual parameters $\left\{a_{i}, b_{i}, c_{i}, t_{m a t, i}\right\}$ in a multivariate normal distribution with parameter 187 mean values $\left\{\bar{a}, \bar{b}, \bar{c}, \bar{t}_{\text {mat }}\right\}$ set as described above and a constant covariance matrix $\Sigma^{2}$. The 188 number of years of the simulated growth curves was also sampled randomly in an uniform 189 distribution $\mathrm{U}(8,18)$ with the constraint that all individuals should have matured at least one 190 year before they die. Four combinations of $\bar{q}$ and $\bar{t}_{\text {mat }}$, resulting in a maturation body mass 191 higher than asymptotic adult body mass, were discarded from the analysis.

192 In order to generate realistic growth curves, the fixed parameters $\left(\bar{a}, \bar{b}+\bar{c}\right.$, and $\left.\Sigma^{2}\right)$ were 193 given the values obtained by applying the method to a real dataset, namely the 1960 cohort of 194 NSSH (see below for details and values). Since $\bar{a}$ and $\bar{b}+\bar{c}$ were fixed, mean asymptotic 195 body mass $\bar{m}_{\infty A}$ was constant across simulated datasets, but the mean growth curve shape 196 changed according to the mean relative investment to reproduction $\bar{q}$ and the mean age at 197 maturation $\bar{t}_{\text {mat }}$ (Fig. 1).

198 The energy based growth model (3) was then fitted to each dataset, and the ability of the 199 method to accurately estimate the parameters was assessed by the following measures (where $200 \wedge$ denotes estimate):

$201 \quad$ - relative error $E_{\bar{x}}=(\hat{\bar{x}}-\bar{x}) / \bar{x}$ and $E_{\sigma_{x}}=\left(\hat{\sigma}_{x}-\sigma_{x}\right) / \sigma_{x}$ measuring the bias on estimates 202 of mean $\bar{x}$ and standard deviation $\sigma_{x}$ of any parameter. 
- absolute error $E_{\rho(x, y)}=\hat{\rho}(x, y)-\rho(x, y)$ for the bias on correlation $\rho(x, y)$ between any pair of parameters $(x, y)$,

- mean of the absolute values of individual relative errors $E_{x}=\left(\sum_{i}\left|\hat{x}_{i}-x_{i}\right| / x_{i}\right) / n$ as a measure of the imprecision of the individual estimates for any parameter $x$ ( $n$ being the number of individuals)

\section{Real datasets}

210 The method was applied to individual growth data for NSP (from IMARES, IJmuiden, 211 Netherlands) and NSSH (from Institute of Marine Research, Bergen, Norway). For NSP, 212 length-at-age data were back-calculated from otoliths sampled from the Dutch commercial catches between 1933 and 1999. Length $l$ was converted into body mass $m$ using the

214 allometric relationship $m=\delta l^{\theta}$ with $\delta=10^{-5} \mathrm{~kg} \cdot \mathrm{cm}^{-1}$ and $\theta=3$ (Rijnsdorp and Storbeck 215 1995). For NSSH, length-at-age data were back-calculated from scales sampled from 216 commercial catches and scientific surveys between 1935 and 1973. These were transformed 217 into body mass using a similar allometric relationship with $\delta=2.32 \times 10^{-5} \mathrm{~kg} \cdot \mathrm{cm}^{-1}$ and $218 \theta=2.81$ (Jennings and Beverton 1991). A description of the data collection and back219 calculation methods including the respective validations can be found in Rijnsdorp et al. 220 (1990) for NSP, and Engelhard et al. (2003) for NSSH. For each stock, two cohorts, one 221 characterized by early maturation and one by late maturation, were chosen to test our method. 222 Only mature females older than 6 years were considered in this study.

223 To assess the validity of our method, estimates (named thereafter growth-based estimates) of 224 age at maturation were compared to independent estimates. For NSP, no independent 225 individual estimate of age at maturation was available and the validity of the method was 226 assessed at the population level. The observed maturity ogive $o_{o}(t)$ - the proportion of 
mature individuals at age $t$ - was calculated for each cohort from commercial catches sampling where individual maturity status was determined by visual inspection of the gonad 229 (from Rijnsdorp and Storbeck (1995)). The growth-based individual estimates of age at 230 maturation $\hat{t}_{\text {mat, } i}$ were used to calculate the probability of maturing at a given age $t$ 231 conditional on being still immature at age $t-1, p(t)$ (i.e. $p(t)=$ number of individuals with $t-1<\hat{t}_{\text {mat, } i}<t$ divided by the number of individuals with $\left.\hat{t}_{\mathrm{mat}, i}>t-1\right)$. Under the assumption 233 that mortality rate does not differ between immature and mature individuals; a growth-based 234 estimate of the maturity ogive, $o_{g}(t)$, was then derived from the probability of maturing at a 235 given age (see Kuparinen et al. (2008) for details) :

$o_{g}(t)=\sum_{\hat{t}_{\mathrm{mat}, i}=1}^{t-1}\left[\left(\prod_{t^{\prime}=0}^{\hat{t}_{\mathrm{mat}, i}-1}\left(1-p\left(t^{\prime}\right)\right)\right) p\left(\hat{t}_{\mathrm{mat}, i}\right)\right]$ for $t>1$

A logistic model was then fitted to the maturity ogives estimated from the sampling of 238 commercial catches and from the growth-based $\hat{t}_{\text {mat }}$ :

$239 o(t)=1 / 1+e^{-(\omega+k t)}$

240 The age at which $50 \%$ of the individuals are mature $A_{50}=-\omega / k$ and slope $k$ of the ogive 241 were used to compare the maturity ogives.

242 For NSSH, the comparison was carried out at the individual level. Winter and summer growth 243 layers on NSSH scales differ between spawning and non-spawning years (Runnström 1936). 244 A direct estimate of age at first spawning was hence 'read' on each individual scale. Since an 245 individual maturing in its $Y^{\text {th }}$ year ( $Y$ being an integer) will reproduce for the first time at age $246 Y+1$, the growth-based estimates of individuals' age at maturation, $\hat{t}_{\text {mat,i }}$, were rounded to the 
next integer to be converted into age at first spawning, $\hat{T}_{\text {mat,i }}$. Growth-based individual

248 estimates $\hat{T}_{\text {mat,i }}$ were then directly compared with scale-based estimates $T_{\text {mat,i }}$.

\section{Results}

\section{Simulated data}

252 Results for the datasets with $m_{0}=10^{-4} \mathrm{~kg}$ are presented first in order to investigate the 253 influence of $\bar{q}$ and $\bar{t}_{\text {mat }}$ on the method's performance. Results for varying values of $m_{0}$ are 254 presented at the end of this section.

\section{Estimation of the multivariate distribution of the life history parameters in the population}

256 The parameter means $\left\{\bar{a}, \bar{b}, \bar{c}, \bar{t}_{\text {mat }}\right\}$ were generally accurately estimated (Fig. 2). Bias for $\bar{a}$

257 and $\bar{t}_{\text {mat }}$ was generally very low with $E_{\bar{a}}$ and $E_{\bar{t}_{m a t}}$ less than 0.01 (but $E_{\bar{a}}=0.06$ for $\bar{q}=4 / 5$ 258 and $\bar{t}_{m a t}=4 \mathrm{yr}$, and $E_{\bar{t}_{m a t}}=-0.09$ for $\bar{q}=1 / 5$ and $\left.\bar{t}_{m a t}=4 \mathrm{yr}\right)$.

259 Bias in estimates of $\bar{b}$ and $\bar{c}$ was moderate with opposite patterns. $\bar{b}$ was well estimated for 260 low $\bar{q}$ and early maturation $\left(\left|E_{\bar{b}}\right| \leq 0.09\right.$ for $\bar{q} \leq 1 / 2$ and $\bar{t}_{\text {mat }} \leq 5 \mathrm{yr}$ ), but under increased $261 \bar{q}$, bias became larger (up to $E_{\bar{b}}=-0.21$ ). In contrast, $E_{\bar{c}}$ was generally positive and lower 262 than 0.10 for moderate to high $\bar{q}$ and late maturation. Bias increased when $\bar{q}$ was low and 263 maturation relatively early (up to $E_{\bar{c}}=0.19$ for $\bar{q}=1 / 5$ and $\bar{t}_{\text {mat }}=6$ yr). The sum $\bar{b}+\bar{c}$, 264 however, was almost always well estimated $\left(\left|E_{\overline{b+c}}\right| \leq 0.05\right)$, suggesting that errors in $\bar{b}$ and $265 \bar{c}$ were compensating each other.

266 Standard deviations of the life history parameters were generally overestimated with bias 267 larger than for their means (Fig. 3; notice the difference of scale between this figure and Fig. 268 2). Bias for $\sigma_{t_{m a t}}$ was low to moderate $\left(E_{\sigma_{t_{m a t}}}\right.$ varying between -0.07 and +0.14$)$. Bias for 
$\sigma_{a}$ was also moderate ( $E_{\sigma_{a}}$ being almost always positive and below 0.15 ) for low $\bar{q}$ and early

270 mean maturation ( $\bar{q} \leq 1 / 2$ and $\left.\bar{t}_{\text {mat }} \leq 6 y r\right)$, but increased for higher $\bar{q}$ and later maturation

271 with $E_{\sigma_{a}}$ between 0.25 and 0.30 . Again, patterns of bias for $\sigma_{b}$ and $\sigma_{c}$ were opposite. For

$272 \bar{q} \leq 1 / 3$, the bias for $\sigma_{b}$ was moderate $\left(\left|E_{\sigma_{b}}\right| \leq 0.17\right)$. However, for higher $\bar{q}$, the bias

273 increased strongly $\left(-0.30 \leq E_{\sigma_{b}} \leq 0.42\right.$ for $\bar{q}$ in $\{1 / 2 ; 2 / 3\}$ and increasing up to 0.70 for

$274 \bar{q}=4 / 5)$. Bias on $\sigma_{c}$ was generally moderate to low $\left(\left|E_{\sigma_{c}}\right| \leq 0.10\right)$, except for low $\bar{q}$ and 275 early maturation $\left(E_{\sigma_{c}}=0.40\right.$ for $\bar{q}=1 / 5$ and $\left.\bar{t}_{\text {mat }}=4 \mathrm{yr}\right)$.

276 The bias in the estimation of correlations between pairs of life history parameters varied 277 according to the parameters involved (Fig. 3; notice that bias on correlation are expressed as 278 absolute errors $)$. Correlations $\rho(a, c), \rho\left(a, t_{\text {mat }}\right)$, and $\rho\left(c, t_{\text {mat }}\right)$ were accurately estimated 279 (errors between -0.15 and +0.15$)$ except under low $\bar{q}(\bar{q}=1 / 5)$ and/or early maturation $280\left(\bar{t}_{\text {mat }}=4 \mathrm{yr}\right)$, when correlation estimates were clearly inaccurate (e.g. $\left.E_{\rho(a, c)}=0.61\right)$. For 281 correlations involving the size-specific maintenance rate $b$, the bias was stronger with errors 282 varying generally between -0.3 and +0.3 with no clear pattern, but rising up to $E_{\rho\left(b, t_{\text {mat }}\right)}=$ 2830.48 and decreasing to $E_{\rho(a, b)}=-0.81$ when $\bar{q}$ was high and mean age at maturation was $284 \operatorname{low}\left(\bar{q}=4 / 5\right.$ and $\left.\bar{t}_{\text {mat }}=4 \mathrm{yr}\right)$.

\section{Estimates of the individual life-history parameters}

286 The accuracy of the individual trait estimates varied across parameters, and, for a given 287 parameter, varied according to the values of $\bar{q}$ and mean age at maturation $\bar{t}_{\text {mat }}$ (Fig. 4). The 288 patterns observed were generally the same as for the bias on the mean parameters. Individual 289 size-specific acquisition rate $a_{i}$ was well estimated, with $E_{a} \leq 0.05$ for all datasets. 290 Estimates of individual age at maturation $\hat{t}_{\text {mat, } i}$ were also very accurate $\left(E_{t_{\text {mat }}}<0.025\right)$, 291 although the error increased moderately (up to $E_{t_{m a t}}=0.10$ ) for low mean age at maturation 
$292\left(\bar{t}_{\text {mat }}=4 \mathrm{yr}\right)$ and low $\bar{q}$. In most cases, the method failed to estimate correctly individual 293 size-specific maintenance and reproductive rates. The error for $b_{i}$ was on average higher than 294 for $c_{i}$. In addition, the errors $E_{b}$ and $E_{c}$ varied in opposite directions with $\bar{q}$. $b_{i}$ was 295 accurately estimated for low $\bar{q}\left(E_{b}<0.08\right.$ for $\left.\bar{q}=1 / 5\right)$, but errors were higher for higher $\bar{q}$, 296 especially at low mean age at maturation (up to $E_{b}=0.40$ for $\bar{q}=4 / 5$ and $\bar{t}_{\text {mat }}=4 \mathrm{yr}$ ). In 297 contrast, $E_{c}$ was low for high $\bar{q}\left(E_{c}<0.08\right.$ for $\bar{q} \geq 2 / 3$, except for $\bar{q}=4 / 5$ and $t_{\text {mat }}=4 y r$ 298 where $E_{c}=0.13$ ) but increased markedly for low $\bar{q}\left(E_{c}\right.$ between 0.11 and 0.19 for $\bar{q} \leq 1 / 3$ ). 299 However, $E_{b}$ and $E_{c}$ compensated so that the sum $E_{b+c}$ was accurately estimated in all cases.

\section{Sensitivity to initial body mass}

301

The method's performance was almost insensitive to the value of initial body mass $m_{0}$. The accuracy of the estimates of parameter means, standard deviations and correlations was similar whatever the value of $m_{0}$ (results not shown). Furthermore, the level of error for individual parameter estimates was hardly affected by the value of $m_{0}$ (Table 1 ).

\section{Real data}

The energy allocation-based growth model fitted very well to the individual growth curves in

307 the four datasets considered $\left(R^{2}>0.98\right.$ in all cases).

308 For both species, estimates of the mean age at maturity $\hat{\bar{t}}_{\text {mat }}$ were different between cohorts, 309 although the confidence intervals largely overlapped for NSP (Table 2 and 3). Estimates of 310 mean size-specific energy allocation rates $\hat{\bar{a}}, \hat{\bar{b}}$ and $\hat{\bar{c}}$ varied between the cohorts (except for $311 \hat{\bar{a}}$ for NSSH). Furthermore, mean size-specific acquisition rate $\hat{\bar{a}}$ was substantially higher in 312 NSP than in NSSH, whereas mean size-specific maintenance $\hat{\bar{b}}$ and reproductive $\hat{\bar{c}}$ rates 313 were relatively similar for the two species. The estimated mean relative reproductive 
314 investment $\hat{\bar{q}}=\hat{\bar{c}} /(\hat{\bar{b}}+\hat{\bar{c}})$ differed between cohorts for NSP (0.24 and 0.63 for the 1963 and 3151969 cohorts respectively) but less for NSSH (0.44 and 0.53 for the 1933 and 1960 cohorts 316 respectively).

317 The correlation structure between parameters was similar both between cohorts and between 318 species (Table 2 and 3). The correlation $\hat{\rho}(a, b)$ was always high and positive and the 319 correlation $\hat{\rho}\left(a, t_{\text {mat }}\right)$ was always negative (although the confidence interval for the 1963 320 cohort for NSP included 0$)$. The correlation $\hat{\rho}\left(c, t_{\text {mat }}\right)$ was always high and negative. The 321 correlations $\hat{\rho}(a, c)$ and $\hat{\rho}\left(b, t_{\text {mat }}\right)$ were almost always weak (expect for the 1960 cohort in $322 \mathrm{NSSH})$. While the correlation $\hat{\rho}(b, c)$ was low for NSSH, high negative values were observed 323 for NSP.

324 The NLME framework allows performing statistical tests on population level characteristics 325 (parameters' means and (co-)variances), which is illustrated here on NSP (Table 2). For the 326 two year-classes of NSP, likelihood ratio tests indicated that the 4 life-history parameters 327 varied significantly among individuals (significant variances, $\mathrm{p}<0.001$ ) and were significantly 328 correlated (significant covariances, $\mathrm{p}<0.001$ ). Furthermore, $F$ tests and a likelihood ratio 329 tests respectively showed that the mean values (fixed effect) of the four traits and the 330 covariance matrix of the random effects differed significantly between the 1963 and 1969 331 cohorts $(\mathrm{p}<0.001$ in all cases).

332 For both cohorts of NSP, the maturity ogives reconstructed from gonad inspection in 333 commercial samples and from the growth-based estimates were very similar (Fig. 5) with very 334 close $A_{50}$ values $\left(A_{50,0,1963}=A_{50, g, 1963}=4.27 y r\right.$ and $A_{50,0,1969}=3.21 y r$ vs. $A_{50, g, 1969}=$ 335 3.00yr where subscripts $o$ refers to maturity status observations and g to growth-based) and 336 rather similar slopes $\left(k_{o, 1963}=1.53\right.$ vs. $k_{g, 1963}=1.11$ and $\left.k_{o, 1969}=k_{g, 1969}=1.71\right)$. For 337 NSSH, the percentage of agreement between the growth-based estimates and the scale-based 338 estimates of individual age at first reproduction were of $61 \%$ and $46 \%$ for cohort 1933 and 
1960, respectively (Table 4). For both cohorts, the agreement was slightly lower for

340 individuals with early maturation (for scale-based age at maturation of 5 years, only $46 \%$ and

$34130 \%$ of agreement) whereas it was much higher for individuals maturing older. For most

342 individuals, the difference between growth-based estimates and scale-based estimates was

343 less than or equal to one year (94\% and 98\% for cohort 1933 and 1960, respectively).

\section{Discussion}

\section{Accuracy of estimates of individual life-history parameters and their population}

\section{distribution}

348 Our study on simulated data indicates that the accuracy of the estimates varies across

349 parameters and according to the species' life-history type. For age at maturation, size-specific energy acquisition rate, and the sum of size-specific maintenance and reproductive rates, population distribution and individual parameters are accurately estimated for all life-history types. In contrast, while they are moderately accurate for the most common life-history strategies, the estimates of size-specific maintenance and reproductive investment rates are clearly poor for extreme life-history types. Size-specific reproductive investment rate is poorly estimated for low reproductive investment because this results in a weaker decrease in somatic growth after maturation (Fig. 1). On the opposite, a high reproductive investment

357 leads to biased estimates of size-specific maintenance rate. This is due to the symmetric roles 358 of these two energy rates in determining asymptotic body mass, $m_{\infty A}=(a /(b+c))^{4}$. 359 Asymptotic body mass and the speed to reach it are estimated accurately, whereas the method 360 fails in distinguishing maintenance from reproduction when imbalance between the two 361 parameters is large. The discrimination ability of the method could be improved by 362 integrating data on reproductive investment. Annual reproductive investment can be assessed 
363 from biological data as the sum of the gonads' energy content before spawning (often

364 measured by proxies, such as the gonado-somatic index) and, if relevant, the energy spent for 365 spawning migration (see Mollet et al. (2010)) for an example on NSP). The energy allocation 366 model could then be fitted to growth and reproductive investment data together by modelling 367 annual reproductive investment as the energy accumulated in the reproductive compartment 368 during one year. However, reproductive investment is generally difficult to measure, 369 especially energy expenditure for spawning migration. Finally, the method was insensitive to 370 the initial body mass value. This supports the choice made here to reduce the number of 371 parameters estimated by assuming a constant initial body mass.

372 The distribution of age at maturation for both cohorts of NSP was accurately estimated by the 373 method, as shown by the high agreement between growth-based and gonad observation based 374 the maturity ogives. For NSSH, estimates of age at first spawning (calculated from the 375 growth-based individual estimates of age at maturation) agreed reasonably well with the scale 376 reading-based ones. The differences between growth-based and scale reading estimates of age 377 at first spawning do not necessarily mean that the growth-based estimates are erroneous, and 378 could also be attributed to errors in scale reading. It is believed that scale estimates are of 379 good quality, but the scale reading method has not been validated, and the level of error or 380 bias is unknown (Baulier and Heino, 2008). Scale readers estimate age at maturation based on 381 the distinction between relatively wide 'coastal' and 'oceanic' rings, corresponding to the 382 immature stage, and narrow 'spawning' rings, corresponding to years after first spawning 383 (Runnström 1936). In some cases, this distinction may be problematic, especially when the 384 last oceanic ring corresponding to the maturation year has an intermediate width (Engelhard 385 et al. 2003). 

history parameters

The application of the method to NSP and NSSH data revealed differences in the life-history parameters between cohorts, in both species. Such variation may result from phenotypic plasticity in response to environmental variations. For instance, energy acquisition is related to food availability (e.g. Kooijman (2000)) which, in turn, depends on the productivity of prey species and on the intensity of the competition for resources. Any variation in environmental factors (e.g. temperature, eutrophication) affecting food productivity, or any change in the abundance of the species competing for the same food, will therefore influence the energy acquisition rate. Changes in temperature also affect the metabolism of individuals, thereby causing variation in size-specific maintenance rate $b$ (Atkinson, 1994).

Temporal variation in energy-based life history traits will result in different growth patterns and, as maturation is closely correlated with growth (Stearns and Koella, 1986), in different timing for the onset of maturation. For instance, the very large 1963 cohort for grew slowly and matured late, probably due to an increased density-dependent competition for food (Rijnsdorp and Van Leeuwen 1992), which also reflects in the lower size-specific acquisition rate for this cohort. Density-dependence may also explain the earlier maturation of the 1960 cohort in NSSH, which grew up in an environment with fewer conspecifics than the 1933 one.

404 The two cohorts being almost 3 decades apart, evolutionary changes might also have 405 happened in response to natural or human-induced selection or both (Engelhard and Heino 406 2004). The higher reproductive investment in the 1960 cohort than in the 1933 one is in agreement with the expectations from fisheries-induced evolution theory. species would provide historical time-series of life-history traits. The analysis of the 
covariation between these time-series and explanatory variables could help to disentangle the

411 respective influence of evolution and phenotypic plasticity.

\section{Comparison with other estimation methods}

413 The first method proposed for estimating age at maturation from individual growth curves

414 was a piecewise linear regression of individual annual body mass increments against body 415 mass to detect the 'breakpoint' corresponding to the decrease in somatic growth occurring 416 after maturation (Rijndorp and Storbeck, 1995). This method was applied recently to Irish Sea 417 plaice (Scott and Heikkonen 2012) and to NSSH (Baulier and Heino 2008). In NSSH a 47.6\% 418 agreement between the 'breakpoint' estimates of age at maturation and those read from scale 419 marks was obtained, with the highest disagreement found for late maturing individuals. 420 Larger error for late maturing individuals may come from the fact that, under the common 421 assumption that maintenance energy scales with a greater power of body mass than energy 422 acquisition $(\beta>\alpha)$, maintenance rate increases with body mass faster than acquisition rate, 423 so that growth rate decreases when body mass exceeds a threshold value $m_{\text {th }}=(b \beta / a \alpha)^{(\beta-\alpha)}$ 424 (from equation (1) when the derivative of growth rate $\partial m_{J} / \partial t$ for $m$ is set to 0 ). If an 425 immature individual reaches $m_{t h}$, the piecewise regression will detect a breakpoint at the age 426 corresponding to $m_{t h}$, which is earlier than maturation. Our method does not suffer from this 427 problem because the underlying energy allocation model integrates explicitly the faster 428 increase of maintenance rate with body mass. In another study, age at maturation in NSSH 429 was estimated from the width of the annual growth layers in scales by discriminant analysis 430 and artificial neural networks resulting in $67 \%$ of agreement with the estimates of age at 431 maturation read in scale marks (Engelhard et al. 2003). However, these methods require to be 432 calibrated using a training dataset consisting of individuals for which the age at maturation is 
433 known. In contrast, the method presented here does not require any a priori knowledge and

434 therefore can be applied to any species for which individual growth trajectories are available.

435 The methods presented in this study and in Mollet et al. (2010) are the first to estimate 436 individual life-history traits simultaneously from individual growth data. These methods 437 accurately estimate the distribution of age at maturation in the population as shown by the 438 comparison of the maturity ogives for NSP in the present study and with maturation reaction 439 norms in Mollet et al. (2010). In the present study, we provide a second validation of the 440 method on real dataset allowing to test the accuracy of the individual age at maturation 441 estimates, by comparing the output of the NLME with estimates read directly from maturation 442 marks on scales for NSSH.

443 The NLME method developed in this paper results in substantial improvements compared to 444 the individual optimisation fit performed in Mollet et al (2010). First, the NLME method 445 reduces the confounding problem between size-specific maintenance and reproductive rates to 446 species with extreme life-history strategies only (see above). The bimodality problem 447 observed in Mollet et al. as a result of confounding is avoided here because the individual 448 parameters are forced to follow a (multivariate) normal distribution. Second, in Mollet et al, 449 the characteristic of the traits distribution in the population, calculated from the individual 450 estimates, are affected by any source of error in the individual estimates (including the 451 bimodality problem, unless a fixed size-specific maintenance rate was assumed). Within the 452 NLME framework, population-level parameters are directly estimated as the fixed effects 453 (mean traits) and (co-)variances between traits, and are independent of the individual 454 estimates of the traits that are calculated a posteriori. Third, the individual optimisation 455 method is over-parameterized (4 parameters are estimated for each individual from only 6 to 45612 growth data points) whereas the NLME method reduces the number of parameters to be 457 estimated to 14 (4 fixed effects and 10 (co-)variances) for the whole dataset thus leading to 
more stable estimates. Finally, the NLME framework is based on a likelihood approach that

459 allows to draw statistical inferences such as testing for differences in the multivariate distribution (means and (co-)variances) of the life-history parameters between distinct groups of individuals (e.g. cohorts) or for the dependence of the population mean traits on explanatory variables (e.g. by including temperature as a covariate; not implemented here).

463 Hence, the NLME method appears more appropriate to characterise the multivariate 464 distribution of life-history parameters in a population and is particularly suitable for evolutionary ecologists who are often more interested in the distribution of traits in a population than in any particular individual values. Although our method may not necessarily provide unbiased estimates of the life history parameters, it provides a consistent interpretation of the data which in many applications matters more.

\section{Limitations and applicability}

470 From a practical point of view, the NLME algorithm requires a sufficiently large sample of 471 individuals (e.g., $\mathrm{n} \geq 30$ ) as well as a sufficiently large number of age-size records per 472 individual, notably during the adult stage, to ensure proper estimation of the decrease in 473 growth rate due to reproductive investment. It may occur that, for a given set of starting 474 values, convergence cannot be reached by the estimation algorithm. It is therefore advised to 475 try various combinations of initial values and test the solutions for their respective goodness 476 of fit and convergence.

477 Given that the performance depends on the life-history strategy, we also recommend, when 478 applying the method to a new species, to evaluate its performance using simulated data in a 479 similar setup as the one presented here.

480 We would also like to remind the reader that the values of the traits estimated by the method 481 are conditional to the value of the scaling exponents of energy rates with body mass. As long 482 as $\alpha<\beta$ and $\alpha<\gamma$, using different exponent value would rescale the estimates of the size- 
specific energy rates, but would not affect estimates of age at maturation. The method would

484 still provide accurate estimates of age at maturation and remain suitable to study the relative differences or changes in the mean and (co-)variances of the other traits.

A potential limitation of our method is that the energy allocation model does not include energy reserves. For NSP for instance, first-time spawners can use all surplus energy for somatic growth because they already have the high energy reserves required for spawning. In contrast, repeat spawners need to rebuild their energy reserves (that are depleted due to spawning the previous year) for the next spawning event before using energy for somatic growth (Rijnsdorp 1990). Hence, first time and repeat spawners start their respective year of reproduction in different energetic conditions, and the growth cost of reproduction may therefore become manifest only from the second spawning year onwards. This could result in an overestimation of age at maturation by our method. Nevertheless, the strong similarity between the growth- and gonad-based maturity ogives observed for NSP suggests that our simplification of energy dynamics has little implications for our primary purpose to estimate age at maturation.

498 Another implicit simplification of the energy allocation model is that the rates of energy 499 acquisition and expenditure are only determined by body mass and not affected by environmental variation, for instance in temperature or food availability, despite substantial empirical evidence (e.g. Brander (1995) or Mollmann et al. (2005)). The effect of neglecting temporal variability in size-specific energy rates might reduce the precision of the estimates 503 and result, if temporal variability is strong, in estimation biases (Mollet et al. 2010). However, 504 the environmental variability levels for which these effects are substantial are unlikely to be 505 common in nature (Mollet et al. 2010).

506 Although individual-level estimates of the parameters may be partly biased in some 507 circumstances, the relatively good accuracy of the estimates of their population means, 
standard deviations and correlations provides potential for a wide range of applications.

509 Firstly, maturity ogives can be calculated from the estimated mean and variance of age at maturation in each cohort. In the context of fish stock assessment for instance, this provides an alternative to the traditional estimation of maturity ogives based on the determination of

512 fish maturity stage by visual inspection of the gonads, which is potentially affected by various 513 sources of errors (see also Scott and Heikkonen (2012)). Moreover, for many fish stocks, 514 maturity data are too scarce to estimate a maturity ogive for each cohort, so that temporal variations in maturity cannot be accounted for. Coupled with automated otolith analyses (Fablet and Le Josse 2004), our method would complement a powerful toolbox to analyse large collections of otoliths and provide historical time series of maturity ogives improving

518 the reconstruction of historical spawning stock biomass time series. The application is not 519 restricted to fish, but can be applied to any other taxa with calcified structures allowing the back-calculation of individual growth trajectories. Our method also provides the potential to study life-history strategies, in particular the phenotypic correlations between life-history traits that can help improve our understanding of life-history trade-offs and intraspecific variation in life-history.

\section{Acknowledgements} providing the data on NSSH. We are also grateful to Mikko Heino and two anonymous reviewers for their critical comments which greatly helped in improving the manuscript. This study was supported by the European research training network FishACE and the strategic research program "Sustainable spatial development of ecosystems, landscapes, seas and 


\section{References}

Atkinson D (1994) Temperature and organism size - a biological law for ectotherms. Advances in Ecological Research 25:1-58

Baulier L, Heino M (2008) Norwegian spring-spawning herring as the test case of piecewise linear regression method for detecting maturation from growth patterns. Journal of Fish Biology 73:2452-2467

Bernardo J (1993) Determinants of maturation in animals. Trends in Ecology and Evolution 8:166-173

Bernardo J (1994) Experimental analysis of allocation in two divergent, natural salamander populations. American Naturalist 143:14-38

Berrigan D, Charnov EL (1994) Reaction norms for age and size at maturity in response to temperature - a puzzle for life historians. Oikos 70:474-478

Brander KM (1995) The effect of temperature on growth of Atlantic cod (Gadus morhua L.). ICES Journal of Marine Science 52:1-10

Campana SE (2001) Accuracy, precision and quality control in age determination, including a review of the use and abuse of age validation methods. Journal of Fish Biology 59:197 $-242$

Dhillon RS, Fox MG (2004) Growth-independent effects of temperature on age and size at maturity in Japanese medaka (Oryzias latipes). Copeia 2004:37-45

Engelhard GH, Dieckmann U, Godø OR (2003) Age at maturation predicted from routine scale measurements in Norwegian spring-spawning herring (Clupea harengus) using discriminant and neural network analyses. ICES Journal of Marine Science 60:304313

Engelhard GH, Heino M (2004) Maturity changes in Norwegian spring-spawning herring Clupea harengus: compensatory or evolutionary responses? Marine Ecology Progress Series 272:245-256

Fablet R, Le Josse N (2004) Automated fish age estimation from otolith images using statistical learning. Fisheries Research 72:279-290

Jennings S, Beverton RJH (1991) Intraspecific variation in the life history tactics of Atlantic herring (Clupea harengus L.) stocks. ICES Journal of Marine Science 48:117-125

Johnson JB (2001) Adaptive life-history evolution in the livebearing fish Brachyrhaphis rhabdophora: Genetic basis for parallel divergence in age and size at maturity and a test of predator-induced plasticity. Evolution 55:1486-1491 
Kooijman SALM (2000) Dynamics enregy and mass budgets in biological systems.

$$
\text { Cambridge University Press, Cambridge, UK }
$$

Kuparinen A, Cano J, Loehr J, Herczeg G, Gonda A, Merila J (2011) Fish age at maturation is influenced by temperature independently of growth. Oecologia Aquatica 167:435-443

Kuparinen A, O’Hara RB, Merilä J (2008) The role of growth history in determining the age and size at maturation. Fish and Fisheries 9:201-207

Mollet FM, Ernande B, Brunel T, Rijnsdorp AD (2010) Multiple growth-related life history traits estimated simultaneously in individuals. Oikos 119:10-26

Mollmann C, Kornilovs G, Fetter M, Koster FW (2005) Climate, zooplankton, and pelagic fish growth in the central Baltic Sea. ICES Journal of Marine Science 62:1270-1280

Pinheiro, J. C. and Bates, D. M. (2000). Mixed Effects Models in S and S-Plus. New York: Springer. 528pp.

Pinheiro J.C., Bates D.M., DebRoy S, Sarkar D, the R Core team (2007) nlme: Linear and Nonlinear Mixed Effects Models. R package version 3.1-86

R Development Core Team (2006) R: A language and environment for statistical computing. R Foundation for Statistical Computing, Vienna, Austria

Reznick DN (1990) Plasticity in age and size at maturity in male guppies (Poecilia reticulata) - an experimental evaluation of alternative models of development. Journal of Evolutionary Biology 3:185-203

Ricklefs RE (2003) Is rate of ontogenetic growth constrained by resource supply or tissue growth potential? A comment on West et al.'s model? Functional Ecology 17:384-393

Rijnsdorp AD (1990) The mecanism of energy allocation over reproduction and somatic growth in the female North Sea plaice, Pleuronectes platessa L. Netherlands journal of Sea Research 25:279-290

Rijnsdorp AD, Storbeck F (1995) Determining the onset of sexual maturity from otoliths of indiviual female North Sea plaice, Pleuronectes platessa L. In: Secor D, Dean J, Campana S (eds) Recent developments in fish otolith research. University of South Carolina Press, Columbia, pp 581-598

Rijnsdorp AD, Van Leeuwen PI (1992) Density dependent and independent changes in somatic growth of female North Sea plaice Pleuronectes platessa between 1930 and 1985 as revealed by back-calculation of otoliths. Marine Ecology Progress Series 88:19-32 
Rijnsdorp AD, van Leeuwen PI, Visser TAM (1990) On the validity and precision of backcalculation of growth form otoliths of the plaice, Pleuronectes platessa L. Fisheries Research 9:97-117

Roff DA (1983) An allocation model of growth and reproduction in fish. Canadian Journal of Fisheries and Aquatic Sciences 40:1395-1404

Runnström S (1936) A study on the life history and migrations of the Norwegian springspawning herring based on the analysis of the winter rings and summer zones of the scales. Fiskeridirektoatets Skrifter, Serie Havundersokelser 5:1-103

Scott RD, Heikkonen J (2012) Estimating age at first maturity in fish from change-points in growth rate. Marine Ecology Progress Series 450:147-157

Stearns SC (1984) The effects of size and phylogeny on patterns of covariation in the life history traits of lizards and snakes. The American Naturalist 123:56-72

Stearns SC, Hoekstra RJ (2005) Evolution. 2nd Ed. Oxford University Press, New York Stearns SC, Koella JC (1986) The evolution of phenotypic plasticity in life-history traits: predictions of reaction norms for age and size at maturity. Evolution 40:893-913 West GB, Brown JH, Enquist BJ (2001) A general model for ontogenetic growth. Nature 413:628-631 


\section{Tables :}

619

620 Table 1. Influence of initial body mass on the accuracy of the individuals' trait estimates. For

621 each value of $m_{0}, \overline{E_{x}}$ is the average of $E_{x}$ (the error on the individual estimates for each 622 parameter $x$ ) over the simulated datasets corresponding to all possible combination of $\bar{q}$ and $623 \bar{t}_{\text {mat }}$.

624

\begin{tabular}{cccccc}
\hline$m_{0}$ & $\overline{E_{a}}$ & $\overline{E_{b}}$ & $\overline{E_{c}}$ & $\overline{E_{b+c}}$ & $\overline{E_{t_{\text {mat }}}}$ \\
\hline $0.5 \times 10^{-4} \mathrm{~kg}$ & 0.052 & 0.213 & 0.141 & 0.065 & 0.027 \\
$1 \times 10^{-4} \mathrm{~kg}$ & 0.047 & 0.249 & 0.144 & 0.059 & 0.024 \\
$1.5 \times 10^{-4} \mathrm{~kg}$ & 0.056 & 0.205 & 0.113 & 0.070 & 0.020 \\
\hline
\end{tabular}

625

626

Table 2. Estimates of the life-history parameters, their correlation matrix and associated

627 statistical tests for two year-classes of NSP (F.E.: fixed effects, sd(R.E): standard deviation of 628 random effects) and their confidence intervals estimated by bootstrap (numbers in brackets).

629 Units are yr for $t_{\mathrm{mat}}, \mathrm{yr}^{-1}$ for $b$ and $c$ and $\mathrm{kg}^{1 / 4} \cdot \mathrm{yr}^{-1}$ for $a$. Hypothesis tests are based on $F$ tests 630 for fixed effects and likelihood ratio tests between nested models that asymptotically follow a $631 \chi^{2}$ under the null hypothesis for random effects' (co-)variances (Pinheiro and Bates 2000).

632

633

\begin{tabular}{lcc}
\hline & & North Sea plaice \\
& YC 1963 & YC 1969 \\
\hline$N$ & 158 & 109 \\
\hline
\end{tabular}

Parameter mean and standard deviation estimates

\begin{tabular}{rrrrr} 
& F.E & sd(R.E) & F.E & sd(R.E) \\
$a$ & $0.87(0.83 ; 0.89)$ & $0.16(0.14 ; 0.18)$ & $0.92(0.86 ; 0.98)$ & $0.19(0.16 ; 0.22)$ \\
$b$ & $0.57(0.42 ; 0.67)$ & $0.23(0.16 ; 0.28)$ & $0.30(0.01 ; 0.45)$ & $0.29(0.01 ; 0.39)$ \\
$c$ & $0.18(0.10 ; 0.29)$ & $0.13(0.10 ; 0.18)$ & $0.53(0.33 ; 0.70)$ & $0.25(0.18 ; 0.32)$ \\
$t_{\text {mat }}$ & $4.76(3.63 ; 6.12)$ & $1.70(1.32 ; 2.14)$ & $3.81(3.35 ; 4.14)$ & $0.99(0.73 ; 1.41)$ \\
\hline
\end{tabular}

\section{Parameter correlation estimates}

$\begin{array}{rrrcrrr} & a & b & c & a & b & c \\ b & 0.72 & & & 0.67 & & \\ & (0.36 ; 0.85) & & & (0.19 ; 0.82) & \end{array}$




\begin{tabular}{crrrrrr}
$c$ & 0.16 & -0.53 & & 0.14 & -0.64 & \\
& $(-0.16 ; 0.59)$ & $(-0.64 ;-0.37)$ & & $(-0.17 ; 0.96)$ & $(-0.76 ;-0.00)$ & -0.80 \\
$t_{\text {mat }}$ & -0.40 & 0.25 & -0.77 & -0.65 & 0.08 & $(-0.92 ;-$ \\
& $(-0.72 ; 0.10)$ & $(-0.18 ; 0.57)$ & $(-0.89 ;-0.51)$ & $(-0.93-;-0.38)$ & $(-0.57 ; 0.47)$ & $0.57)$ \\
\hline
\end{tabular}

\section{Statistical tests :}

The traits are variable among individuals ${ }^{1}$

$$
\chi_{4}^{2}=2150, \mathrm{p}<0.001 \quad \begin{gathered}
\chi_{4}^{2}=360 \\
\mathrm{p}<0.001
\end{gathered}
$$

The traits are correlated ${ }^{2}$ :

$$
\chi_{6}^{2}=442, \mathrm{p}<0.001 \quad \begin{gathered}
\chi_{6}^{2}=418, \\
\mathrm{p}<0.001
\end{gathered}
$$

Difference in traits' mean values between the two $\mathrm{YC}^{3}$ :

$$
\begin{array}{cc}
a & F_{2009}^{1}=30468, \mathrm{p}<0.001 \\
b & F_{2009}^{1}=1838, \mathrm{p}<0.001 \\
c & F_{2009}^{1}=524, \mathrm{p}<0.001 \\
t_{\text {mat }} & F_{2009}^{1}=256, \mathrm{p}<0.001
\end{array}
$$

Difference in traits covariance between the two $\mathrm{YC}^{4}: \chi_{10}^{2}=57, \mathrm{p}<0.001$

$634{ }^{1}$ : likelihood ratio test comparing the fit of a model with a diagonal covariance matrix for the 635 random effects and a model with no random effects (separately for each YC).

$636 \quad 2$ : likelihood ratio test comparing the fit of a model with a symmetric co-variance matrix for the 637 random effects and a model with a diagonal co-variance matrix (separately for each YC)

$638{ }^{3}: F$ tests of the YC effect coded as a factor acting linearly on the fixed part of parameters

$639 \boldsymbol{\mu} \sim \boldsymbol{\mu}_{0}+\boldsymbol{\mu}_{1} \cdot Y C$ in a model fitted on the two YC data pooled.

$640{ }^{4}$ : likelihood ratio test comparing the fit of a model on the two YC data pooled with a) a

641 different random-effect co-variance matrix for each YC, and b) a similar co-variance matrix for 642 the two YC. 
644 Table 3. Estimates of the life history parameters and their correlation matrix for two year645 classes of NSSH (F.E.: fixed effects, sd(R.E): standard deviation of random effects). Units are $646 \mathrm{yr}$ for $t_{\mathrm{mat}}, \mathrm{yr}^{-1}$ for $b$ and $c$ and $\mathrm{kg}^{1 / 4} \cdot \mathrm{yr}^{-1}$ for $a$.

647

648

\begin{tabular}{ccc}
\hline & \multicolumn{2}{c}{ Norwegian spring spawning herring } \\
YC 1933 & YC 1960 \\
\hline$N$ & 142 & 261 \\
\hline
\end{tabular}

Parameter mean and standard deviation estimates

\begin{tabular}{rrrrrrr}
\multicolumn{1}{c}{ F.E } & sd(R.E) & F.E & sd(R.E) \\
$a$ & 0.50 & 0.15 & 0.51 & 0.08 & \\
$b$ & 0.42 & 0.18 & 0.28 & 0.08 & \\
$c$ & 0.20 & 0.07 & 0.32 & 0.07 & \\
$t_{\text {mat }}$ & 6.32 & 1.19 & 5.51 & 0.60 & \\
\hline \multicolumn{7}{c}{ Parameter correlation estimates } \\
$b$ & 0.87 & $b$ & $c$ & $a$ & $b$ & $c$ \\
$c$ & 0.13 & -0.36 & & 0.73 & & \\
$t_{\text {mat }}$ & -0.34 & 0.15 & -0.94 & -0.76 & 0.13 & \\
\hline
\end{tabular}

649

650 
651 Table 4 : Agreement between the growth based $\left(\hat{T}_{\text {mat,i }}\right)$ and scales based $\left(T_{\text {mat,i }}\right)$ estimates of 652 age at first reproduction for NSSH.

\begin{tabular}{crrrrrrrr}
\hline & \multicolumn{3}{c}{ Year-class 1933 } & & \multicolumn{3}{c}{ Year-class 1960 } \\
\cline { 2 - 4 } \cline { 6 - 8 } Age & $\mathrm{n}$ & Correct & Deviation $\leq 1$ & & $\mathrm{n}$ & Correct & Deviation $\leq 1$ \\
\hline 4 & 1 & $100 \%$ & $100 \%$ & & & & \\
5 & 13 & $46 \%$ & $100 \%$ & & 122 & $30 \%$ & $100 \%$ \\
6 & 33 & $67 \%$ & $97 \%$ & & 104 & $61 \%$ & $97 \%$ \\
4 & 49 & $55 \%$ & $92 \%$ & & 35 & $54 \%$ & $97 \%$ \\
8 & 45 & $67 \%$ & $93 \%$ & & & & \\
9 & 1 & $100 \%$ & $100 \%$ & & & & \\
All ages & 142 & $61 \%$ & $94 \%$ & & 261 & $46 \%$ & $98 \%$
\end{tabular}

653

654

655 


\section{Figure legends}

657

658

659

660

661

662

663

664

665

666

667

668

669

670

671

672

673

674

675

676

677

678

Fig. 1 : Growth curves for varying relative reproductive investment $q(q=c /(b+c)$, where $b$ and $c$ are size-specific maintenance and reproductive rates respectively) with fixed age at maturation, $t_{\mathrm{mat}}=6$ years, and fixed adult asymptotic size, $m_{\infty A}=0.423 \mathrm{~kg}$

Fig. 2: Bias in the estimation of mean a. size-specific energy acquisition ( $(\hat{\bar{a}})$, b-d. maintenance $(\hat{\bar{b}})$ and reproductive investment $(\hat{\bar{c}})$ rates, and e. mean age at maturation $\left(\hat{\bar{t}}_{\text {mat }}\right)$, from simulated growth data in relation to the true values of mean relative reproductive investment $\bar{q}$ and mean age at maturation $\bar{t}_{\text {mat }}$ used in the data simulation. Bias is expressed as the relative error $E_{\bar{x}}$ between the estimated and the true mean of each parameter (see Material and Methods section). White and black disks represent negative and positive bias respectively; size of the symbols indicates scale

Fig. 3 : Bias in the estimation of standard deviations for a. size-specific energy acquisition $\left(\sigma_{a}\right)$, c. maintenance $\left(\sigma_{b}\right)$ and $\mathbf{f}$. reproductive investment $\left(\sigma_{c}\right)$ rates, and $\mathbf{j}$. age at maturation $\left(\sigma_{t_{\text {mat }}}\right)$ and b.,d.,e.,g.,h.,i. correlations between these traits $(\rho)$ from simulated growth data in relation to the true values of mean relative reproductive investment $\bar{q}$ and mean age at maturation $\bar{t}_{\text {mat }}$ used in the data simulation. Bias on standard deviation is expressed as the relative error $E_{\sigma_{x}}$ (see Material and Methods section); bias on the correlations is expressed as the absolute difference between the estimated and true value 
679 Fig. 4 : Error in the estimation of the individual size-specific a. energy acquisition ( $\left.\hat{a}_{i}\right)$, b.-d.

680 maintenance $\left(\hat{b}_{i}\right)$ and reproductive investment $\left(\hat{c}_{i}\right)$ rates and e. age at maturation $\left(\hat{t}_{\text {mat, } i}\right)$

681 from simulated growth data in relation to the true values of mean relative reproductive

682 investment $\bar{q}$ and mean age at maturation $\bar{t}_{\text {mat }}$ used in the data simulation. Error is

683 expressed as the mean of the absolute values of individual relative errors $E_{x}$ (see

$684 \quad$ Material and Methods section)

685

686 Fig. 5 : Maturity ogives calculated from gonad observations in catch samples (triangles) and 687 from individual age at maturation estimated from back-calculated growth curves 688 (circles) with the corresponding fitted logistic models (dashed and solid lines 689 respectively) for the 1963 (filled symbols) and 1969 (empty symbols) year-classes of $690 \quad$ North Sea plaice

691 
692

693

694

695

696

697

698

699

700

701

702

703

704

705

706

707

708

709

710

711

712

713

714

715

716

717

718

Fig. 1 Brunel et al.

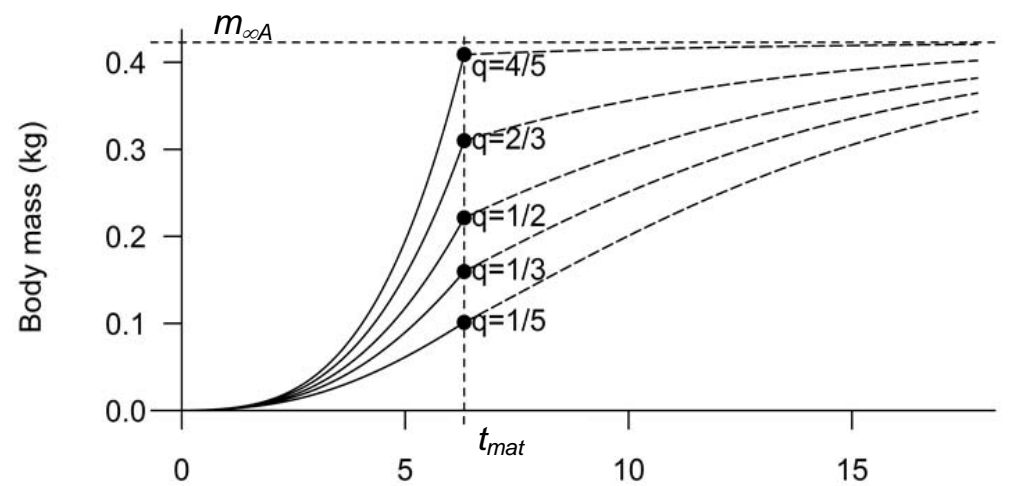

Age (years)

719

720

721

722

723

724

725

726

727

728 
731

732

a. Error on $\bar{a}$

740

741

742

745

746

747

749

750

751

752

753
743

744

748

Fig. 2. Brunel et al.

d. Error on $\bar{b}+\bar{c}$

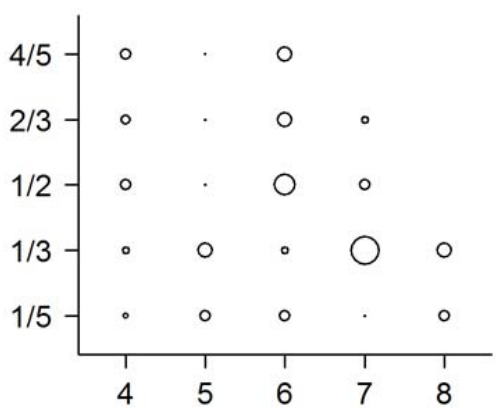

754
Mean age at maturation, $\bar{t}_{\text {mat }}$ b. Error on $\bar{b}$

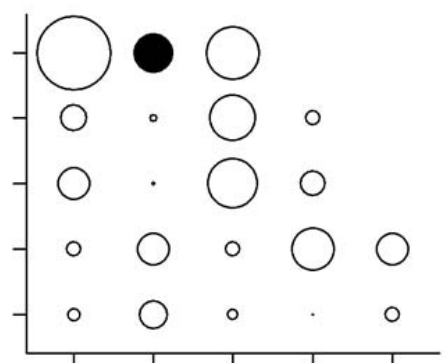

e. Error on $\bar{t}_{m a t}$

c. Error on $\bar{c}$
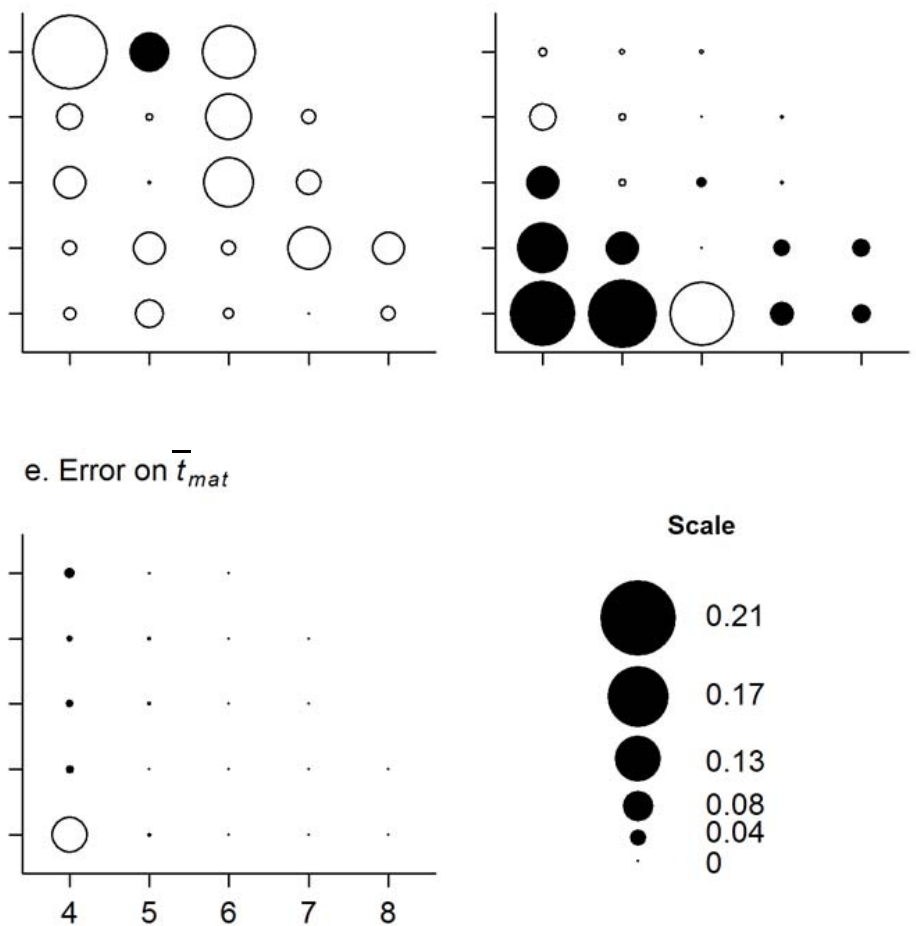

Scale

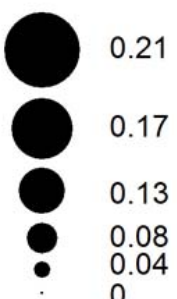


a. Error on $\sigma_{a}$

761

762

763

764

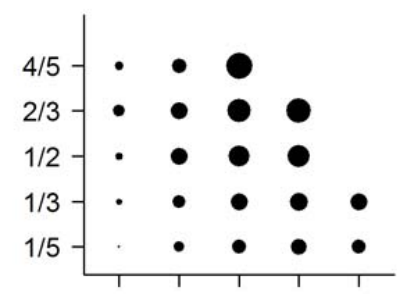

765

766

b. Error on $\rho(a, b) \quad$ c. Error on $\sigma_{b}$

767

768

769

770

771

772

773

774

775

776
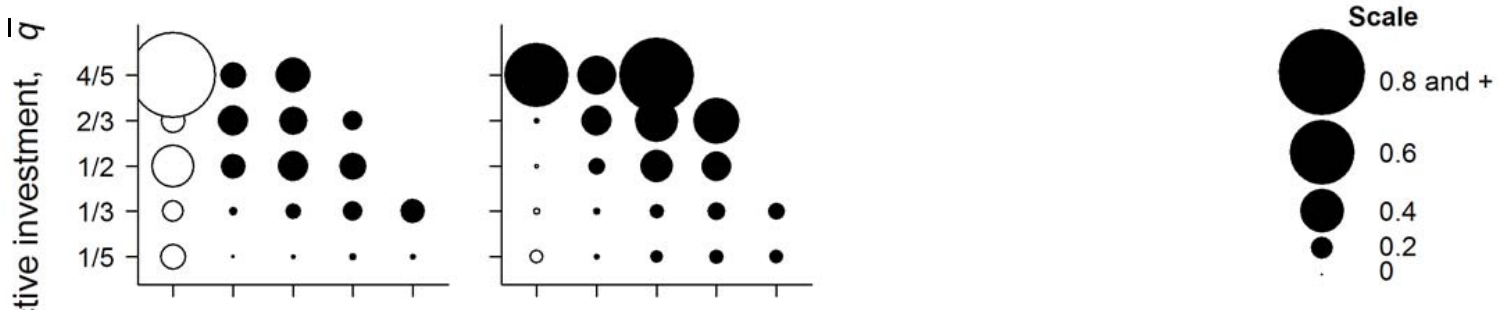

777

778

779

780

781

782

783

\section{d. Error on $\rho(a, c)$}

e. Error on $\rho(b, c)$

f. Error on $\sigma_{c}$
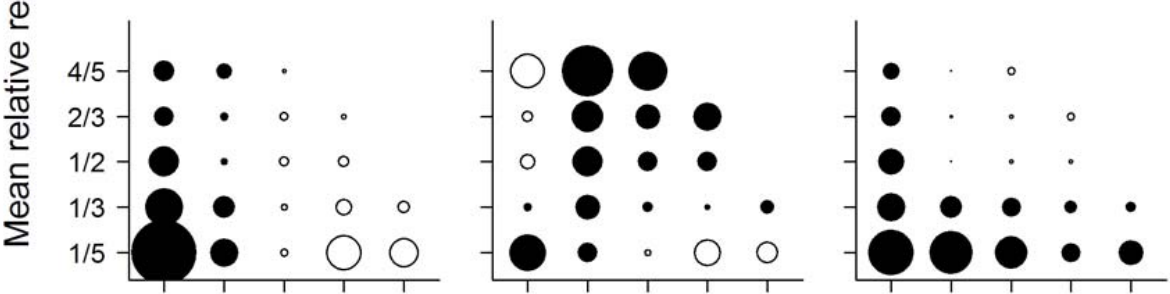

\section{g. Error on $\rho\left(a, t_{m a t}\right)$}

h. Error on $\rho\left(b, t_{\text {mat }}\right)$

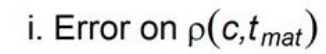

j. Error on $\sigma_{t_{\text {mat }}}$
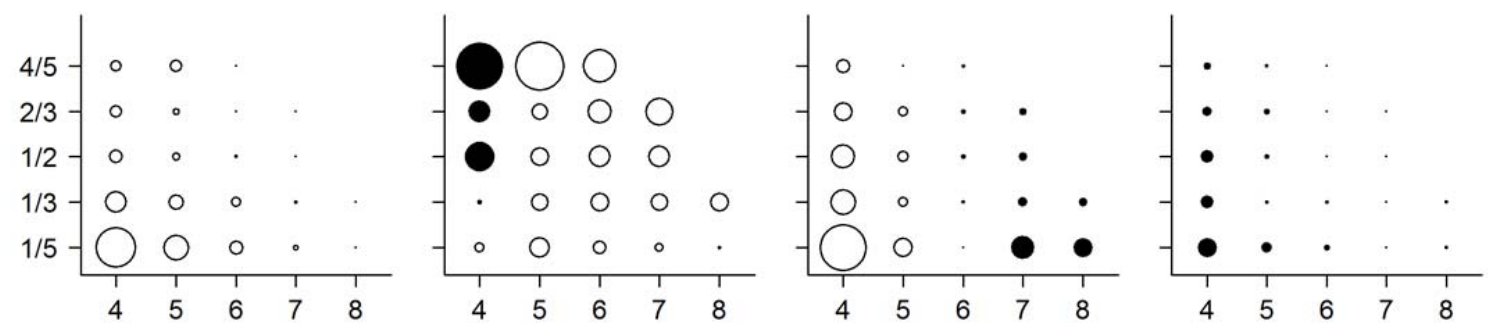

784

Mean age at maturation, $\bar{t}_{\text {mat }}$

785

786

787

Fig. 3. Brunel et al.

788 
791
a. Error on $a_{i}$

792

793

794

795

796

797

798

799

800

801

802

803

804

805

806

807

808 Fig. 4. Brunel et al.

809

810

811 b. Error on $b_{i}$

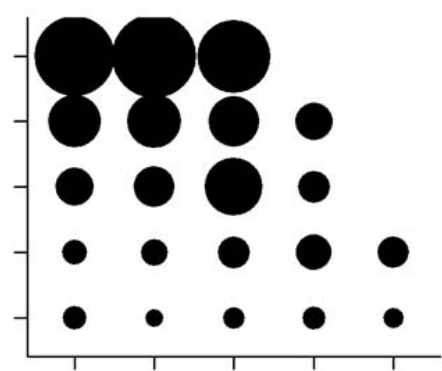

e. Error on $t_{m a t_{i}}$

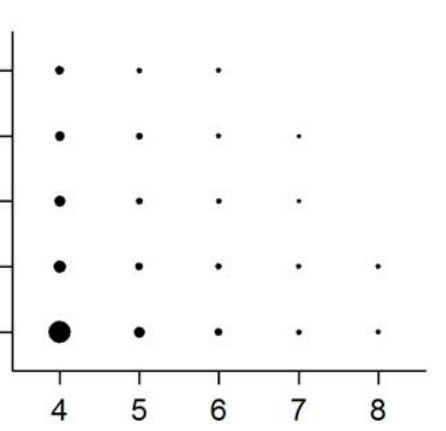

Mean age at maturation, $\bar{t}_{\text {mat }}$ c. Error on $c_{i}$
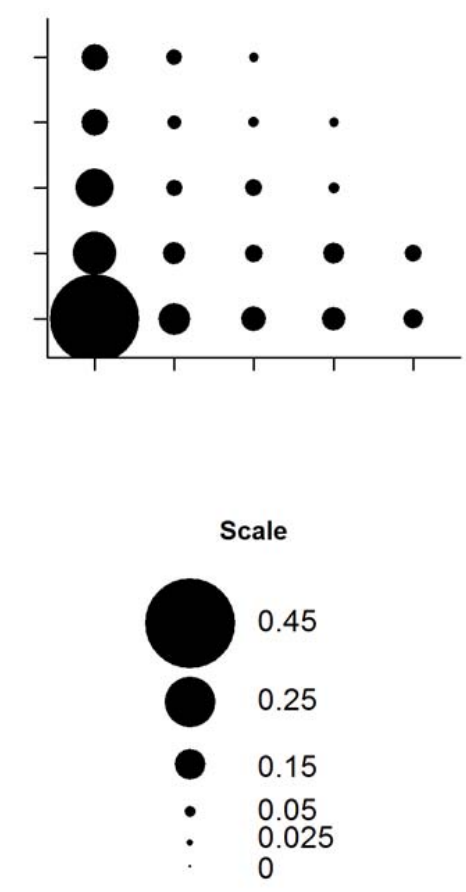
812

813

814

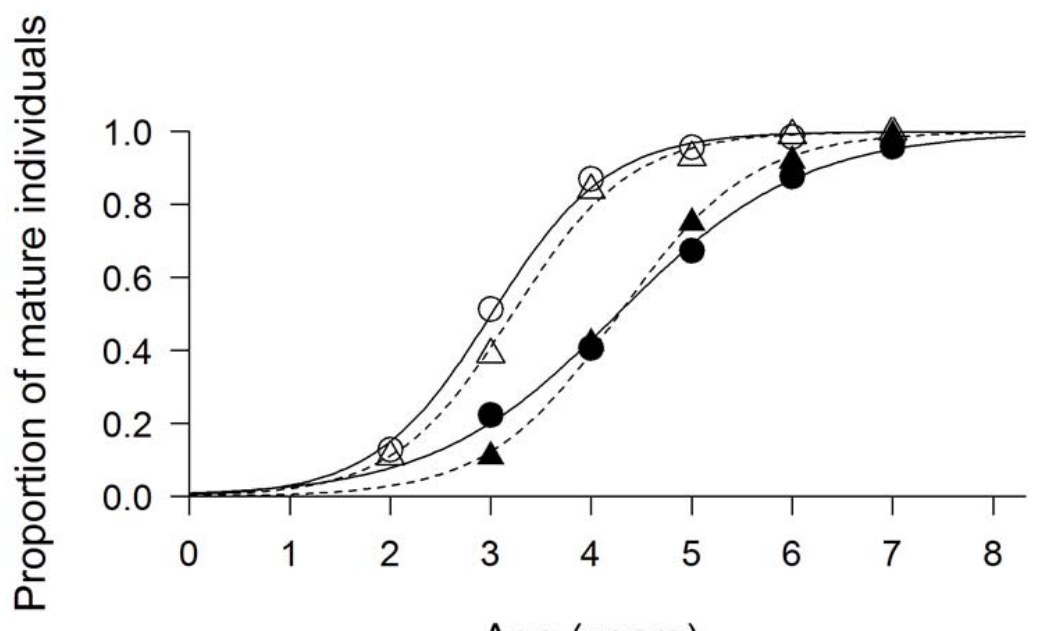

815

Age (years)

816

817

818

819

820 Fig. 5. Brunel et al.

821

822

823 
Supplementary material for

Estimating age at maturation and energy-based life-history traits from individual growth trajectories with nonlinear mixed-effects models

by

Thomas Brunel, Bruno Ernande, Fabian Mollet and Adriaan D. Rijnsdorp

\section{Supplementary material S 1:}

\section{Energy allocation-based growth model}

Among all classes of energy allocation-based growth models, a frequently used differential equation for somatic growth, known as the Pütter balance equation (Pütter (1920) as cited in Ricklefs (2003)), has the following general form:

$d m / d t=a m^{\alpha}-b m^{\beta} \quad$ for $t \leq t_{m a t}$

$d m / d t=a m^{\alpha}-b m^{\beta}-c m^{\gamma} \quad$ for $t>t_{m a t}$

where $m$ is body mass, $t$ is time, $t_{\text {mat }}$ is age at maturation and the coefficients $a, b$ and $c$ are the size-specific rates of energy acquisition, energy use for body maintenance, and energy use for reproduction, respectively. According to this model, the amount of energy assimilated is used simultaneously for body maintenance, somatic growth and, after maturation, reproduction (figure $\mathrm{S} 1$ ). The relative importance of energy flows to maintenance, somatic growth and reproduction varies during the individual's lifetime, as $m$ increases, depending on the value of exponents $\alpha, \beta$ and $\gamma$.

Several versions of this equation differing in these exponents values have been proposed in the literature (see e.g. Table 2 in Ricklefs (2003)). Among the most popular ones are those proposed by Von Bertalanffy ( $\alpha=2 / 3$ and $\beta=\gamma=1$ ) (von Bertalanffy 1957) and West et al. ( $\alpha=3 / 4$ and $\beta=\gamma=1$ ) (West et al. 2001). In von Bertalanffy's equation, the rate of energy acquisition is proportional to body surface which scales with $m^{2 / 3}$, whereas in West et al.'s model, the rate of energy acquisition is assumed to be limited by the size of the capillary network that brings energy to cells, which has a fractal form and scales with $m^{3 / 4}$. In our study, for the purpose of illustration we use the exponent values from West et al. because it has been intensively used in recent life history studies (Charnov et al. 2001; Economo et al. 2005).

The value of the scaling exponents of metabolic rates with body mass has been subject to considerable debates on both conceptual and empirical grounds (Allen and Gillooly 2007; O'Connor et al. 2007; Ricklefs 2003), the scaling power $\alpha$ of energy acquisition rate being the main point of controversy. However, using $\alpha=2 / 3$ or $\alpha=3 / 4$ results in very similar growth curves and performances in terms of goodness of fit (Banavar et al. 2002) and the results of 
our study are almost unchanged whatever the exponent values chosen among these options. For NSP for instance, fitting the 1963 cohort data with $\alpha=2 / 3$ gave similar estimates of age at maturation than the one presented in table 1, and, although they were of course rescaled, the estimates of size-specific energy rates correlated very well with those obtained with $\alpha=3 / 4$ (results not shown).

In practice, as soon as maintenance and reproductive energy rates scale with a greater power of body mass than energy acquisition rate, i.e. $\beta>\alpha$ and $\gamma>\alpha$ (eq. (S1b)), the results of our study are almost unchanged whatever the actual exponent values. An ad hoc alternative to conceptual exponent values would consist in estimating empirically exponent values for each dataset considered. Among 26 fish species, empirical estimates of $\alpha$ range from 0.66 to 0.80 with mean 0.69 (Hanson et al. 1999), lying thus in between the most popular conceptual values: $\alpha=2 / 3$ (von Bertalanffy 1957) and $\alpha=3 / 4$ (West et al. 2001). For NSSH, no estimate was available in the literature, but for NSP, Fonds et al. (1992) found $\alpha=0.78$, which is close to the value we used $\alpha=3 / 4$.

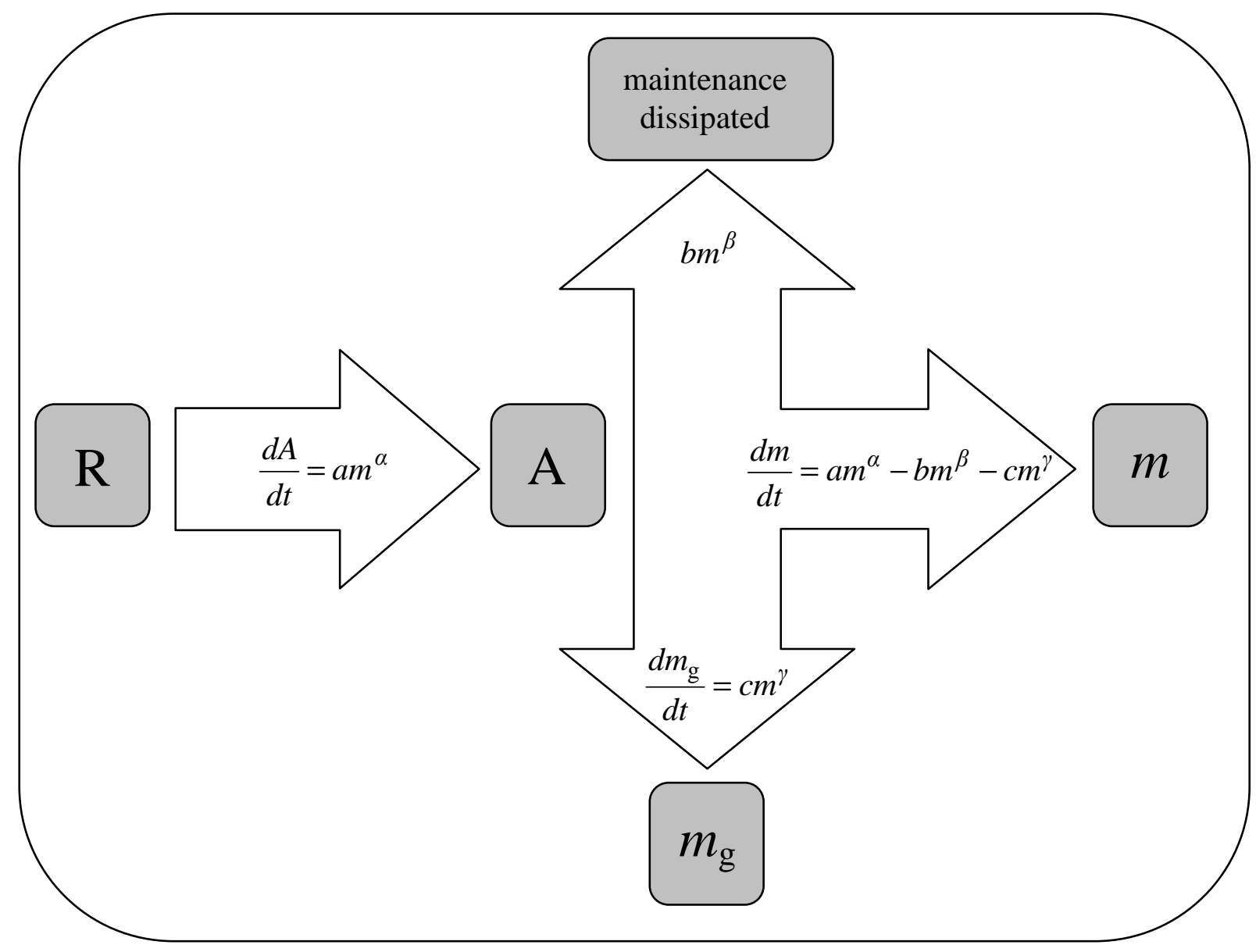

Figure S1 : Diagram of energy allocation in an adult individual according to Pütter balance equations, with $\mathrm{R}$ being resources in mass equivalent, A total acquired energy in mass equivalent, $m_{g}$ gonad mass and $m$ somatic mass. The rates of energy acquisition and 
energy use by the three compartments (somatic mass, maintenance and gonad mass) are given by the equations in each arrows. 


\section{References}

Allen AP, Gillooly JF (2007) The mechanistic basis of the metabolic theory of ecology. Oikos 116:1073-1077

Banavar JR, Damuth J, Maritan A, Rinaldo A (2002) Modelling universality and scaling. Nature 420:626

Charnov EL, Turner JH, Winemiller KO (2001) Reproductive constraints and the evolution of life histories with indeterminate growth. Proc. Natl. Acad. Sci. USA 98:9460-9464

Economo EP, Kerkhoff AJ, Enquist BJ (2005) Allometric growth, life-history invariants and population energetics. Ecol. Lett. 8:353-360

Fonds M, Cronie R, Vethaak AD, Van Der Puyl P (1992) Metabolism, food consumption and growth of plaice (Pleuronectes platessa) and flounder (Platichthys flesus) in relation to fish size and temperature. Neth J Sea Res 29:127-143

Hanson PC, Johnson TB, Schindler DE, Kitchell DE (1999) Fish bioenergetics 3.0. University of Wisconsin Seagrant Institute, Madison, WI

O'Connor MP et al. (2007) Reconsidering the mechanistic basis of the metabolic theory of ecology. Oikos 116:1058-1072

Pütter A (1920) Studien über Physiologische Ähnlichkeit. VI. Wachstumsähnlichkeiten. Pflügers Archive für Gesamte Physiologie Menschen und Tiere 180:298-340

Ricklefs RE (2003) Is rate of ontogenetic growth constrained by resource supply or tissue growth potential? A comment on West et al. 's model? Funct. Ecol 17:384-393

von Bertalanffy L (1957) Quantitative laws in metabolism and growth. Q. Rev. Biol 32:217231

West GB, Brown JH, Enquist BJ (2001) A general model for ontogenetic growth. Nature 413:628-631 


\section{Supplementary material S 2:}

\section{Fitting the growth model using nonlinear mixed effect modelling}

NLME models are commonly used for the analysis of longitudinal data (repeated measures over time on the same individuals), and to perform individual fits of a nonlinear parametric function for a set of individuals at once. In the present case, the formulation of the NLME model is the following one, with bold denoting vector and matrices:

$$
m_{i, t}=m\left(t, \phi_{i}\right)+\varepsilon_{i, t}
$$

where $m_{i, t}$ is the body mass of the $i^{\text {th }}$ individual at age $t, m\left(t, \boldsymbol{\phi}_{i}\right)$ is the growth model given by equation (3) with $\phi_{i}=\left(a_{i}, b_{i}, c_{i}, t_{\mathrm{mat}, i}\right)^{\mathrm{T}}$ the vector of parameters to be estimated for the $i^{\text {th }}$ individual, and $\varepsilon_{i, t}$ is a normally distributed error term.

NLME models estimate the vector of parameters $\phi_{i}$ for each individual $i$ as the sum of a vector of fixed effects $\boldsymbol{\mu}=\left(\bar{a}, \bar{b}, \bar{c}, \bar{t}_{\text {mat }}\right)^{\mathrm{T}}-$ the population means of the parameters - and a vector of random effects $\boldsymbol{\eta}_{i}=\left(\Delta a_{i}, \Delta b_{i}, \Delta c_{i}, \Delta t_{\text {mat }, i}\right)-$ the deviations of individual parameters from their population means:

$$
\phi_{i}=\mu+\eta_{\mathrm{i}} \text { where } \eta_{\mathrm{i}} \sim \operatorname{MVN}\left(\mathbf{0}, \Sigma^{2}\right)
$$

where MVN refers to multivariate normal distribution. The random effects are constrained to follow a multivariate normal distribution $\operatorname{MVN}\left(\mathbf{0}, \Sigma^{2}\right)$ centered on $\mathbf{0}$, since they describe deviation from population means, with a variance-covariance matrix $\Sigma^{2}$. In other terms, parameters of each individual growth curve are estimated given the 'average' population growth curve and assuming they are normally distributed in the population.

NLME models were fitted using the nlme package (Pinheiro et al. 2007) of the statistical software R (R Development Core Team 2006). Current algorithms have poor convergence properties when estimating more than 4 parameters. Therefore, we limited the number of estimated parameters to 4 . To this end, for both the simulated and real datasets, the growth model was lagged by one year by replacing $m_{0}$ by $m_{1}$ and $t$ by $t-1$ in equation (2) and $m_{1}$ was set constant at its mean value in the data.

The algorithm estimates the fixed effect parameters $\boldsymbol{\mu}$ using an iterative procedure that minimizes penalized nonlinear least squares, which requires providing starting values. The nonlinear least-square estimates of $\bar{a}, \bar{b}, \bar{c}$ and $\bar{t}_{\text {mat }}$ obtained by fitting the energy allocation model to the whole dataset considered (nls function, $\mathrm{R}$ base statistical software) were then used as starting values. As the confidence intervals produced by the nlme package are based on an approximation of the variance-covariance matrix of the parameter estimates obtained from the likelihood function, they are regarded as uncertain, and bootstrapping is considered more appropriate (Pinheiro and Bates, 2000). As bootstrapping (1000 replicates) combined to 
fitting NLME is computationally very intensive, confidence intervals were produced only for NSP. For further details on NLME models and the fitting procedure used, the reader can refer to (Lindstrom and Bates 1990).

The NLME framework allows us to perform statistical tests on population level characteristics (parameters' means and (co-)variances),. Hypothesis tests are based on $F$ tests for fixed effects and likelihood ratio tests between nested models that asymptotically follow a $\chi^{2}$ under the null hypothesis for random effects' (co-)variances (Pinheiro and Bates 2000).

Lindstrom MJ, Bates DM (1990) Nonlinear Mixed Effects Models for Repeated Measures Data. Biometrics 46:673-687

Pinheiro J, Bates D, DebRoy S, Sarkar D, the R Core team (2007) nlme: Linear and Nonlinear Mixed Effects Models. R package version 3.1-86

R Development Core Team (2006) R: A language and environment for statistical computing. R Foundation for Statistical Computing, Vienna, Austria 